\title{
Exposition périnatale aux analogues nucléosidiques et dysfonctionnement mitochondrial persistant
}

a tolérance du traitement antiviral administré durant la grossesse pour la prévention de la transmission mère-enfant du VIH a d'emblée été - et reste - une préoccupation. Ce traitement, administré durant la seconde moitié de la grossesse, pendant le travail et chez le nouveau-né durant 6 semaines a démontré son efficacité dans une étude contrôlée contre placebo (protocole ACTG076) puisque le taux de transmission était réduit de $67 \%$ [1]. A la suite des recommandations officielles de mise en place de cette prévention, une importante réduction du nombre d'enfants infectés en période périnatale a été observée dans toutes les cohortes épidémiologiques concernées [2]. Plusieurs programmes de traitement préventif ont été mis en place dans les pays en voie de développement où vivent la plupart des femmes infectées par le VIH. Les premières analyses de toxicité sont plutôt rassurantes [3]. Le principal effet secondaire, qui était prévisible, est une anémie macrocytaire qui se corrige rapidement après l'arrêt du traitement. On ne peut toutefois exclure qu'un effet indésirable non repéré jusqu'à présent s'exprime tardivement, après les quelques mois de suivi usuel des nouveau-nés non infectés par le VIH. Cela a justifié la mise en place d'études de suivi à long terme de nouveau-nés exposés au traitement antiviral (étude ACTG219 par exemple) ou de registres nationaux des prescriptions d'antirétroviraux durant la grossesse, base d'un travail de pharmacovigilance.
Notre attention a été récemment attirée par huit observations de dysfonctionnement mitochondrial chez des enfants non infectés par le VIH, suivis dans le cadre de la cohorte périnatale française [4]. La fréquence des maladies mitochondriales constitutionnelles dans la population générale, est mal connue [5-7], mais on peut l'estimer à 1/5000 à 1/20 000 enfants.

Trois de ces enfants avaient une symptomatologie neurologique sévère - s'apparentant pour deux au cadre général du syndrome d'Alpers, pour l'autre au syndrome de Leigh. Deux d'entre eux sont décédés. Un quatrième avait une symptomatologie neurologique moins sévère, constituée d'un syndrome pyramidal discret et de convulsions hyperthermiques répétées. Un cinquième avait un tableau associant une myopathie modérée, une cardiomyopathie et une hyperlactatémie persistante. Enfin, trois enfants ne présentaient que des perturbations biologiques, détectées dans le cadre du suivi usuel des enfants nés de mère infectée par le VIH : élévation persistante des transaminases, de la lipase et/ou des lactates sanguins. Aucun de ces huit enfants n'était infecté par le VIH. Le traitement préventif administré durant la grossesse était 4 fois la zidovudine, 4 fois l'association zidovudine-lamivudine. Aucune des mères n'était toxicomane. Le profil enzymatique constaté pour ces cinq enfants symptomatiques affirmait sans ambiguité un dysfonctionnement mitochondrial portant sur le complexe I et parfois IV de la chaîne respiratoire. Le déficit était partiel pour les enfants moins sévè- rement atteints. L'étude de l'ADN mitochondrial ne retrouvait pas les délétions, duplications ni mutations classiquement associées aux encéphalopathies mitochondriales constitutionnelles, notamment celles du syndrome neuropathy, ataxia and retinitis pigmentosa (NARP)-Leigh. Il est important de noter toutefois qu'une majorité de ces maladies mitochondriales constitutionnelles ne sont pas encore caractérisées sur le plan génomique.

La toxicité mitochondriale des analogues nucléosidiques anti-VIH est bien connue chez l'adulte $(\mathrm{m} / \mathrm{s} 1991$, $\left.n^{\circ} 5, p .521\right)$ et l'enfant infecté par le VIH (pour revues, voir [8-10]). Elle est principalement liée à l'inhibition par les nucléosides de la v-polymérase, responsable de la duplication de l'ADN mitochondrial. En outre, les analogues nucléosidiques antirétroviraux actuellement utilisés appartiennent au groupe des 2', 3'-didésoxy-analogues et sont dépourvus d'un radical hydroxyle en position 3 '; ils agissent donc aussi en interrompant la chaîne d'ADN dans laquelle ils sont intégrés. De nombreux modèles expérimentaux cellulaires ou animaux confirment ces données. La traduction clinique diffère toutefois selon les molécules antivirales utilisées: celle de la zidovudine est principalement hématologique et musculaire incluant des cardiomyopathies chez l'enfant. Celle de la zalcitabine, de la stavudine, de la didanosine s'exprime surtout par des neuropathies périphériques mais des pancréatites et des hépatites ont aussi été décrites avec les deux dernières. La zidovudine, la stavudine, la 
didanosine peuvent aussi entraîner une acidose lactique sévère. Paradoxalement, la toxicité mitochondriale de la lamivudine semble moindre que celle des autres molécules. La lamivudine est, in vitro, la molécule qui interagit le moins avec la v-polymérase. La mauvaise pénétration de sa forme 5' triphosphate dans la mitochondrie en même temps que son rôle de substrat dans l'activité 3'-5' exonuclease de la $v$ polymérase rendrait son intégration à l'ADN mitochondrial moins facile. Son implication dans plusieurs observations de pancréatite chez l'enfant contraste avec cette relative innocuité in vitro. Aucune observation d'encéphalopathie démyélinisante mitochondriale d'origine toxique n'a été à ce jour rapportée chez l'adulte ou l'enfant traité au long cours par ces nucléosides. Le diagnostic en serait difficile dans le contexte de l'infection par le VIH dont on connaît le neurotropisme en même temps que l'association à certaines infections opportunistes démyélinisantes du système nerveux central. Plusieurs modèles cellulaires et animaux illustrent la toxicité potentielle sur le système nerveux périphérique de certaines de ces molécules.

Exceptée l'anémie, dont la physiopathologie est peut être multifactorielle, aucune toxicité mitochondriale n'a encore été décrite chez le fotus et les nouveau-nés exposés in utero aux analogues nucléosidiques. Plusieurs résultats du groupe de $\mathrm{M}$. Poirier du National Cancer Institute (NCI) ont toutefois attiré récemment l'attention sur ce problème potentiel [11, 12]. Après avoir démontré l'intégration de la zidovudine dans l'ADN mitochondrial de fotus de singe erythrocebus patas après exposition transplacentaire, ils démontrent - dans ce même modèle animal - un dysfonctionnement enzymatique mitochondrial. Pour une exposition à des doses et sur des durées semblables à celles utilisées pour les femmes infectées par le VIH-1, une diminution des complexes I de la chaîne respiratoire en même temps qu'une morphologie anormale des mitochondries en microscopie électronique est mise en évidence dans le muscle squelettique et cardiaque des fœetus. On ne peut dire dans ce modèle si cette toxicité est réversible après la naissance, ni quelle serait sa traduction clinique.

L'hypothèse d'une toxicité médicamenteuse est donc extrêmement probable mais devra être formellement démontrée par la poursuite de l'analyse épidémiologique en cours et de l'approche expérimentale sur l'animal. Cette hypothèse incite à évoquer de principe un tel dysfonctionnement mitochondrial devant des symptômes inexpliqués chez un enfant "exposé » au traitement antirétroviral en période périnatale. Le diagnostic de mitochondriopathie n'est pas toujours aisé et requiert le plus souvent des investigations agressives. La traduction clinique - comme pour les huit enfants ici décrits - est extrêmement variable, pouvant toucher de nombreux organes. Il est en outre envisageable, mais cela reste à démontrer, qu'une toxicité $a$ minima, sans traduction clinique et réversible après l'arrêt du traitement, puisse être engendrée. Les observations récentes d'acidose lactique transitoire réversible à l'arrêt du traitement vont dans ce sens [13]. Une analyse prospective autant que rétrospective, centrée sur la symptomatologie clinique et biologique mitochondriale, est en cours tant pour l'exposition in utero à la zidovudine en monothérapie que pour toutes les associations nucléosidiques. Il est possible que cette toxicité s'exerce sur un terrain génétique particulier qu'il conviendra de déterminer, comme cela a pu l'être pour d'autres substances tel que le valproate [14] ou les aminoglycosides [15].

Dans l'état actuel de nos connaissances, ces données ne remettent bien sûr pas en cause le principe fondamental de la prophylaxie de la transmission périnatale du VIH. La situation devient plus complexe depuis l'avènement des associations antirétrovirales, qu'il s'agisse du maintien d'un traitement efficace débuté avant la grossesse ou de la volonté d'utiliser un traitement préventif à l'action antivirale plus puissante que celle de la zido- vudine en monothérapie. Actuellement, plus de la moitié des femmes reçoivent ainsi une multithérapie durant la grossesse. Ces données incitent toutefois à une certaine prudence, à un effort d'information des femmes sur les incertitudes qui pèsent encore sur ce type de traitement parallèlement à leur extraordinaire efficacité à protéger l'enfant d'une maladie potentiellement mortelle.

\section{Stéphane Blanche}

Service d'immunologie-hématologie pédiatrique et Inserm U.429, Développement normal et pathologique du système immunitaire, Hôpital Necker, 149, rue de Sèvres, 75743 Paris, Cedex 15, France.

\section{Marc Tardieu}

Service de neurologie pédiatrique et Inserm CRI 9612, Virus, neurone et immunité, Hôpital de Bicêtre, 78, rue du Général-Leclerc, 94276 Le Kremlin Bicêtre, France.

\section{Pierre Rustin}

Inserm U. 393, Handicaps génétiques de l'enfant, Hôpital Necker, 149, rue de Sèvres, 75743 Paris Cedex 15, France.

\section{RÉFÉRENCES}

1. Connor EM, Sperling RS, Gelber R, et al. Reduction of maternal infant transmission of human immunodeficiency virus type 1 with zidovudine treatment. $N$ Engl J Med $1994 ; 331$ : 1173-80.

2. Mayaux MI, Teglas JP, Mandelbrot L, et al. Acceptability and impact of zidovudine prevention on mother to child HIV-1 transmission in France. J Pediatr 1997; 131: 85762.

3. Sperling RS, Shapiro DE, McSherry GD, et al. Safety of the maternal-infant zidovudine regimen utilized in the Pediatric AIDS Clinical Trial Group 076 Study. AIDS 1998; 12: 1805-13.

4. Blanche S, Tardieu M, Rustin P, et al. Persistent mitochondrial dysfunction and perinatal exposure to antiretroviral analogues. Lancet 1999; 354: 1084-9. 


\section{RÉFÉRENCES}

5. Johns DR. Mitochondrial DNA and disease. N Engl J Med 1995; 333: 638-45.

6. Poulton J. Mitochondrial gene mutations. Eur J Paed Neurol 1998; 2: 99-103.

7. Di Mauro S, Moares CT. Mitochondrial encephalomyopathies. Arch Neurol 1993; 50 : 1197-208.

8. Swartz MN. Mitochondrial toxicity New adverse drugs effects. N Engl J Med 1995; 333: 1146-8.

9. Lewis W, Dalakas MC. Mitochondrial toxicity of antiviral drugs. Nat Med 1995; 1 : 417-21.

10. Brinkman K, Hofstede HJM, Burger DM, Smeitink JAM, Koopmans PP. Adverse effects of reverse transcriptase inhibitors: mitochondrial toxicity as common pathway. AIDS 1998; 12: 1735-44.

11. Olivero OA, Anderson LM, Diwan BA, et al. Transplacental effects of 3'-azido-2', 3'dideoxythymidine (AZT): tumorigenicity in mice and genotoxicity in mice and monkeys. J Natl Cancer Inst 1997; 89 : 1602-8.

12. Gerschenson M, Erhart SW, Paik CY, Poirier MC. NCI, NIH, Bethesda, MD. Transplacental exposure to 3'-azido-2', 3'dideoxythymidine (AZT) is genotoxic to fetal skeletal and cardiac muscle mitochondria. Am J Hum Gen 1998; 63: 928.

13. Scalfaro P, Chesaux IJ, Buchwalder PA, et al. Severe transient neonatal lactic acidosis during prophylactic zidovudine treatment. Intensive Care Med 1998; 24 : 247-50.

14. Ponchaut S, Vanhoof f, Veitch K. Valproate and cytochrome c oxidase deficiency. Eur J Pediatr 1995; 154 : 133-5.

15. Fischel-Ghodsian N. Mitochondrial mutations and hearing loss: paradigm for mitochondrial genetics. Am J Hum Genet $1998 ; 62: 15-9$

TIRÉS À PART

S. Blanche.

\section{4-26 mai 2000}

Saint-Cloud/Paris

France

ORGANISATION

Centre de Biogéographie-Écologie BIOGÉO

UMR 8505 CNRS/ENS Fontenay/Saint-Cloud Le Parc

92211 SAINT-CLOUD/France

(C) 33 (0) 141123500

ธ 33 (0) 141123540

e-mail : biogeo@ens-flc.fr

Comité d'organisation

assuré par les membres suivants de BIOGÉO :

Marie-Odile BOISSIER, Micheline HOTYAT, Jean-Paul OUDINET, Bertrand SAJALOLI, Yolène THOMAS

Responsable : Stanislas WICHEREK

Secrétariat : Jérôme BAUSSART 